\title{
20 years of Tm:Ho:YLF and LuLF Laser Development for Global Winds Measurements
}

\author{
Upendra N. Singh, Brian M. Walsh, Jirong Yu, Mulugeta Petros, Michael J. Kavaya, Norman P. Barnes \\ NASA Langley Research Center, Hampton, VA 23681 \\ upendra.n.singh@nasa.gov
}

\begin{abstract}
NASA Langley Research Center has a long history of developing $2 \mu \mathrm{m}$ lasers. From fundamental spectroscopy research, theoretical prediction of new materials, laser demonstration and engineering of lidar systems, it has been a very successful program spanning around two decades. This article covers the program development from the early research to the present instrumentation. A brief historical perspective of Tm:Ho work by early researchers is also given. OCIS codes: (140.0140) Lasers and laser optics; (160.0160) Materials; (280.0280) Remote sensing and sensors
\end{abstract}

\section{Historical retrospective}

The National Aeronautics and Space administration (NASA) has been performing ground, airborne, and space-based scientific measurements since it was formed in 1958 after the National Advisory Committee for Aeronautics (NACA) was dissolved and it's resources transferred to the newly created NASA. Initial ground and airborne measurements were made with in situ instruments. By necessity, initial earth observation space-based missions were accomplished with passive remote sensing. Eventually, a new technology, active optical radar, light detection and ranging, or lidar, matured to the point that it was conceivable to consider active remote sensing using lidar for space missions. Part of the motivation for consideration of lidar was due to the tremendous progresses made in solid-state lasers fueled by advances in crystal growth quality and pump laser diode technology. This is especially true for $2 \mu \mathrm{m}$ lasers using Tm:Ho materials. $2 \mu \mathrm{m}$ lasers are ideally suited for mid infrared remote sensing to monitor the atmosphere of Planet Earth and for exploration on other planets. Such $2 \mu \mathrm{m}$ lasers can be used directly for the remote sensing of wind using heterodyne technology and both water and carbon dioxide using DIAL technology.

In 1965, researchers at Bell Labs produced the first Tm:Ho co-doped laser, an $\alpha \beta-Y A G$ operating at $2.1 \mu \mathrm{m}$. This was a CW tungsten lamp pumped system operating at 77K. In 1971 researchers at Sanders Associates produced an $\alpha \beta$-YLF operating at $2.06 \mu \mathrm{m}$. This was a flashlamp-pumped pulsed laser, also operating at $77 \mathrm{~K}$. The first room temperature Tm:Ho laser was demonstrated by researchers at the Universitat Hamburg in 1985. This was a CW Krypton-pumped laser operating at $2.1 \mu \mathrm{m}$ in Cr:Tm:Ho:YSAG and Cr:Tm:Ho:YSGG. The commercial availability of diode lasers in the 1980's was a perfect match for Tm:Ho solid-state lasers due to the good overlap of diode laser wavelengths with $\mathrm{Tm}$ absorption. The first experiments utilizing diode pumping of Tm:Ho materials were done at the Naval Research Laboratory in 1986-87. They demonstrated diode-pumped $2 \mu \mathrm{m}$ laser action in Tm:Ho:YAG and Tm:Ho:YLF. This led shortly thereafter to the first eye-safe coherent lidar in 1991. This lidar system was based on a diode pumped Tm:Ho:YAG at $2.1 \mu \mathrm{m}$. It is important to acknowledge the early pioneering research in this field.

\section{Early work at NASA Langley}

Fundamental spectroscopic work on Tm:Ho laser materials at NASA Langley Research Center (LaRC) began in the late 1980's and continued into the early 1990's. The motivation was to completely characterize these materials in the spectroscopy and gain a deeper understanding of the physics involved, which could be used to advantage in the 2$\mu \mathrm{m}$ laser development. The work was not only based in experiment, but theory was used as well. The theoretical work included using a quantum mechanical model to predict spectroscopic parameters difficult to measure, such as those involving energy transfer processes, but also to predict new materials and assess their laser potential. Laser models were also utilized to project performance parameters. The outcome of this work was a fairly complete understanding of Tm:Ho systems and the prediction that LuAG and LuLF offered advantages over YAG and YLF, respectively. By 1997 NASA LaRC had demonstrated a room-temperature, Tm:Ho:YLF laser amplifier generating $700 \mathrm{~mJ}$ at $2 \mu \mathrm{m}$ from a $50 \mathrm{~mJ}$ flashlamp pumped laser. In 1998, Injection-seeded, room-temperature, diode-pumped Ho:Tm:YLF laser with output energy of $600 \mathrm{~mJ}$ at $10 \mathrm{~Hz}$ was demonstrated. In 2002-2003 a double pulse $600 \mathrm{~mJ}$ $\mathrm{Tm}:$ Ho YLF laser/amplifier was demonstrated. The Tm:Ho technology reached impressive performance levels in 2006 when researchers at NASA LaRC produced 1 Joule per pulse at $2.06 \mu \mathrm{m}$ in Tm:Ho:LuLF, a material invented and developed at NASA Langley, as previously mentioned. All of this development from fundamental physics to engineering $2 \mu \mathrm{m}$ Tm:Ho lasers set the stage for the development of state-of-the-art, eye-safe, compact, lidar systems that could be used for airborne or space-based remote sensing measurements [1-4]. 


\section{Motivation and mission requirements}

In the US, NASA, NOAA and DOD researchers have been working to enable 3-D winds space missions since the 1970s. The over 40 years of work towards the global winds mission in the US has included over 50 studies, encompassing theoretical development, computer simulation of the wind measurement technique and of the utility of the wind measurements, measurement requirements development, space mission design, lidar technology development, and ground and airborne validation. NASA and NOAA scientists have worked with lidar scientists to formulate the wind measurement requirements appropriately stated for a lidar solution.

There is a consensus among researchers that the final operational wind sensor should be a hybrid pulsed Doppler wind profiling lidar with scanning ability or multiple fixed views. The term hybrid refers to the complementary, simultaneous wind measurement by both a coherent-detection and direct-detection lidar. Conceptually, the coherent lidar uses aerosol particles for its signal and favors the lower altitudes, while the direct lidar uses molecules for its signal and favors higher altitudes. The US National Research Council's advice to NASA [5] recently endorsed both the global winds mission and the hybrid lidar concept.

The logical flow of requirements at NASA is from societal benefit of measurement to mission to instrument. Here we discuss the requirements on the coherent Doppler lidar system portion of the hybrid Doppler lidar instrument. The requirements for laser include:

- Pulse energy E $>0.25 \mathrm{~J} /$ pulse

- $\quad$ Pulse rate $(\mathrm{PR})=10 \mathrm{~Hz}$

- $\quad$ Excellent beam quality, $\mathrm{M}^{2}<1.2$

- $\quad$ Single frequency pulse with near transform limited spectrum, minimal chirp

- $\quad>100$ ns pulse duration with $>175$ ns desired

- Conductively cooled laser heads and pump laser diode arrays (LDAs)

- Laser wall plug efficiency (WPE) $>1.2 \%$ (not including control electronics, heat removal, or seed laser)

- Laser lifetime $>630 \mathrm{M}$ shots for initial 2-year mission

During the $20+$ yearlong time, the requirements on the pulsed laser parameters and their priorities changed slightly due to wisdom gained in mission studies. This refers to the relative priorities of mass, volume, electrical efficiency, beam quality, spectral purity, and shot lifetime. We utilized interim stages in the technology development path for ground and airborne validation, and for various science applications. This reveals how we pursued multiple threads of laser development to meet the various requirements.

\section{Instrument development}

As noted in the requirements, a conductively-cooled laser transmitter with diode pumping is needed for space operation, as liquid cooling is active cooling as opposed to passive cooling provided by conductively cooled system. Fig. 1 presents selected photographs documenting the laser cooling head development.

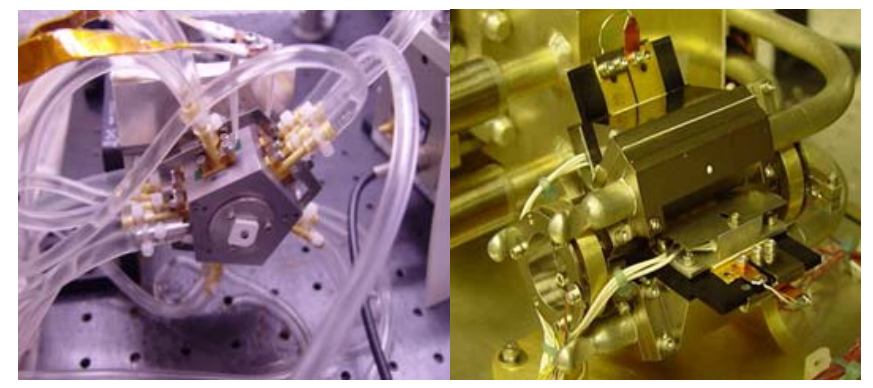

Figure 1: (Left): Early version of laser head containing laser rod and pump LDAs and 22 water channels. (Right) Conductively-cooled laser head no water channels

In 2007, efforts at NASA LaRC split into two parallel paths, both of which were an outcome of the previously described Tm:Ho:YLF and LuLF laser work, outlined in section 2. The liquid cooled (LC) version of the laser was utilized to pursue a Science/liquid cooled (LC) track for measurements through developing coherent Doppler lidar systems in ground and airborne deployment. In parallel LaRC worked on the Technology/conductively cooled (CC) track, also for developing coherent Doppler lidar, but for space deployment. The instruments for these two tracks are shown in Fig 2. The reminder of the article will focus on the Technology (CC) track. Work on a CC version of the 
laser began in 2003 to further advance the laser technology to space readiness. A fully CC laser oscillator with 3sided diode pumping geometry was designed, fabricated and demonstrated in 2004 at NASA LaRC. The laser performance matched the $100 \mathrm{~mJ}$ energy output of the LC laser oscillator. Subsequently, a CC amplifier laser head with 5-sided diode pumping geometry was completed in 2005 with record energy output of $400 \mathrm{~mJ}$ in a double pass amplifier configuration. NASA LaRC designed and fabricated CC oscillator and amplifier modules with heat pipes. However, the lasers demonstrated during this effort were not hardened and ruggedized for deployment and would require several development steps to reach the maturity required to be included in a space-based wind measurement. This motivated an Innovative Partnership Program (IPP) effort, jointly funded by NASA LaRC, Earth Science Division at NASA HQ, and Fibertek in 2007 to develop a conductively-cooled Tm:Ho:LuLF 2-micron laser. NASA LaRC partnered with Fibertek on the IPP and transferred the 2-micron laser technology knowledge to them. The IPP targeted construction of a $2 \mu \mathrm{m}$ Risk Reduction Laser Transmitter (RRLT) in the form of a hardened, "space-like" breadboard laser meeting NASA's optical performance goals and demonstrating the shot lifetime required for spacebased operation. In addition, it would be used to identify the path forward for the next iteration of the technology maturation. The IPP program concluded in 2011 with the delivery of a $200 \mathrm{~mJ}, 5 \mathrm{~Hz}$ packaged laser.
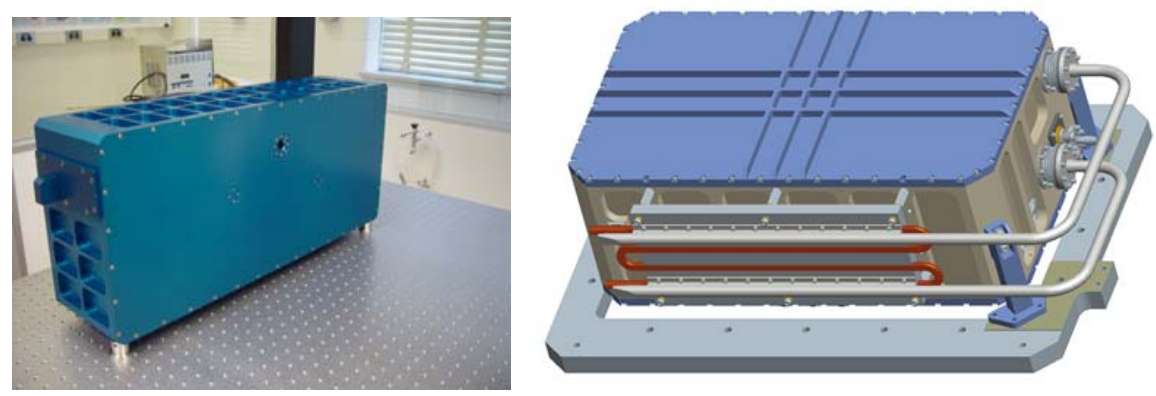

Figure 2: (Left): Liquid cooled Doppler wind lidar transmitter for ground and airborne deployment (Right) Conductively cooled Doppler wind lidar transmitter for space deployment

\section{Current and future development}

In 2012, NASA LaRC and Fibertek entered into a partnership to demonstrate a hardened solid state 2-micron laser in a compact package on a program funded through the NASA Advanced Component Technology (ACT) program under the Earth Science Technology Office (ESTO). Under this program, effort is ongoing towards building a compact, fully conductively cooled, single frequency, space hardened, electrically efficient, $2.053 \mu \mathrm{m}$ $\mathrm{Tm}$ :Ho; LuLF laser delivering $250 \mathrm{~mJ}$ at $10 \mathrm{~Hz}$ with $\mathrm{M}^{2}<2$. This laser system will meet the requirements for the space-based 3-D wind measurement from Earth orbit.

\section{Acknowledgements}

The authors will like to acknowledge the funding and support from NASA Earth Science Technology Office (ESTO) and Science Mission Directorate at NASA headquarters. Authors would also like to acknowledge numerous technical contributors from university, industry, in particular Fibertek Inc, and the government during last 20 years.

\section{References}

[1] B.M. Walsh, N.P. Barnes, M. Petros, J. Yu, and U.N. Singh, "Spectroscopy and modeling of solid state lanthanide lasers: Application to trivalent $\mathrm{Tm}^{3+}$ and $\mathrm{Ho}^{3+}$ in $\mathrm{YLiF}_{4}$ and $\mathrm{LuLiF}_{4}$," Journal of Applied Physics 95, 3255-3271 (2004).

[2] J. Yu, B.C. Trieu, E.A. Modlin, U.N. Singh, M.J. Kavaya, S. Chen, Y. Bai, P.J. Petzar, and M. Petros, "1 J/pulse Q-switched $2 \mu \mathrm{m}$ solid state laser," Optics Letters 31, 462-464 (2006).

[3] U. N. Singh, S. Ismail, M. J. Kavaya, D. M. Winker, and F. Amzajerdian, "Space-Based Lidar," Chapter 9 in "Laser Remote Sensing," Takashi Fujii and Tetsuo Fukuchi, editors, CRC Press, Optical Science and Engineering Series, Vol. 97, pp. 781-881, ISBN 0824742567,2005

[4] U. N. Singh, J. Yu, M. Petros, S. Chen, M. J. Kavaya, B. C. Trieu, Y. Bai, P. J. Petzar, E. A. Modlin, G. J. Koch and J. Y. Beyon, “Advances in High Energy Solid-State 2-micron Laser Transmitter Development for Ground and Airborne Wind and CO2 Measurements" Invited Paper, Lidar Technologies, Techniques, and Measurements for Atmospheric Remote Sensing VI (edited by Upendra N. Singh and Gelsomina Pappalardo), Proc. of SPIE, Vol. 7832, pp7832202-1 - 783202-18, ISBN 978-0-8194-8349-2 (2010)

[5] National Research Council (NRC), "Earth Science and Applications from Space: National Imperatives for the Next Decade and Beyond," The National Academies Press, Wash DC 2005, (Jan. 2007). Note: This is also sometimes known as the "Earth Science Decadal Survey". 